\title{
STATIONARY VECTOR MEASURES AND POSITIVE DEFINITE TRANSLATION INVARIANT BIMEASURES
}

\author{
HANNU NIEMI
}

Introduction. Let $G$ be a locally compact Abelian group and let $\Gamma$ be its dual group.

We present a Bochner representation theorem for positive definite translation invariant bimeasures defined on $G \times G$. In fact, we show that a mapping $B: \mathscr{K}_{C}(G) \times \mathscr{K}_{C}(G) \rightarrow C$ is a positive definite translation invariant bimeasure, if and only if there exists a uniquely determined positive Radon measure $v: \mathscr{K}_{C}(\Gamma) \rightarrow C$ such that $\mathscr{F F} f \overline{\mathscr{F} g} \in \mathscr{L}^{1}(v)$ and

$$
\text { (*) } \quad B(f, \bar{g})=\int \mathscr{\mathscr { F }} f \overline{\mathscr{F} g} d v \text { for all } f, g \in \mathscr{K}_{C}(G) \text {. }
$$

Furthermore, we present a spectral representation theorem for stationary vector measures. In other words, we show that a mapping $\mu: \mathscr{K}_{C}(G) \rightarrow H$, with values in a Hilbert space $H$, is a stationary vector measure, if and only if there exists a uniquely determined orthogonally scattered vector measure $\mu_{0}: \mathscr{K}_{C}(\Gamma) \rightarrow H$ such that $\mathscr{F} f \in \mathscr{L}^{1}\left(\mu_{0}\right)$ and

$$
\text { (**) } \quad \mu(f)=\int \mathscr{F} f d \mu_{0} \text { for all } f \in \mathscr{K}_{C}(G) \text {. }
$$

The representation (*) is closely related to $(* *)$. If $\mu: \mathscr{K}_{C}(G) \rightarrow H$ is a stationary vector measure, which has the representation $(* *)$ and if (the positive definite translation invariant) bimeasure $B(f, g)=(\mu(f) \mid \mu(\bar{g})), f, g \in \mathscr{K}_{C}(G)$, has the representation $(*)$, then

$$
\left(\mu_{0}(\varphi) \mid \mu_{0}(\psi)\right)=v(\varphi \bar{\psi}) \text { for all } \varphi, \psi \in \mathscr{K}_{C}(\Gamma) .
$$

The representation (*) is a generalization of a representation theorem for positive definite Radon measures on $G$ due to Godement (cf. Argabright and Gil de Lamadrid [1; Theorem 4.1], Berg [4], [5], Berg and Forst [6; Theorem 4.5] and Godement [11]); and it is closely related to the well-known Bochner-Schwarz representation theorem for translation invariant positive definite distributions (cf. Gelfand and Vilenkin [9; pp. 166-169]). On the other hand, the representation (**) is closely related to the spectral representation for second order stationary random distributions (cf. Itô [13] or Gelfand and Vilenkin [9; p. 271]). 
This paper is closely related to the works of Berg [4], [5], Berg and Forst [6; pp. 17-26] and Argabright and Gil de Lamadrid [1]. However, one of the main motivations to this work is to present an approach to the spectral analysis of stationary vector measures, which is completely free from the use of distribution theory (cf. Brillinger [7], Daley and Vere-Jones [8] and the references given there).

1. Stationary vector measures. In this section we present some preliminary results concerning stationary vector measures defined on a locally compact Abelian group.

Let $F$ be a locally compact Abelian group (or a locally compact Hausdorff space). By $\mathscr{K}_{C}(F)$ we denote the linear space of all continuous complex-valued functions $f$ defined on $F$ with compact support supp $f$. Let $K$ be a compact subset in $F$. By $\mathscr{K}_{C}(F ; K)$ we denote the linear space of all $f \in \mathscr{K}_{C}(F)$ for which supp $f \subset K$. The topology of $\mathscr{K}_{C}(F ; K)$ is defined by the supremum norm. The topology of $\mathscr{K}_{C}(F)$ is defined to be the locally convex inductive limit of the Banach spaces $\mathscr{K}_{C}(F ; L)$ with respect to the canonical injections $j_{L}: \mathscr{K}_{C}(F ; L) \rightarrow \mathscr{K}_{C}(F) ; L \subset F, L$ compact.

Let $H$ be a complex Hilbert space. Recall that a continuous linear mapping $\mu: \mathscr{K}_{C}(F) \rightarrow H$ is a vector measure defined on $F$ with values in $H$. A vector measure $\mu: \mathscr{K}_{C}(F) \rightarrow H$ is bounded, if the linear mapping $\mu: \mathscr{K}_{C}(F) \rightarrow H$ is continuous when the space $\mathscr{K}_{C}(F)$ carries the topology defined by the supremum norm.

Let $\mu$ be a vector measure with values in $H$ (or a Radon measure) defined on $F$. By $\mathscr{L}^{p}(\mu)$ we denote the set of all functions $u: F \rightarrow C$ for which $|u|^{p}$ is $\mu$-integrable $p=1$, 2. (In this paper we apply the integration theory for vector measures introduced by Thomas [18].)

Let $E$ be a normed space. It follows from the reflexivity of a Hilbert space that all bounded linear mappings $A: E \rightarrow H$ are weakly compact. Therefore, for every vector measure (resp. bounded vector measure) $\mu: \mathscr{K}_{C}(F) \rightarrow H$ all the bounded Borel functions with compact support (resp. all bounded Borel functions) are $\mu$-integrable [18; pp. 86-87 and p. 101].

Let $\mu: \mathscr{K}_{C}(F) \rightarrow H$ be a vector measure. In what follows we use the notation $\operatorname{sp}\{\mu\}=\mu\left(\mathscr{K}_{C}(F)\right)$; and by $\overline{\mathrm{sp}}\{\mu\}$ we denote the closure of $\operatorname{sp}\{\mu\}$ in $H$. Recall that

[18; p. 69].

$$
\int u d \mu \in \overline{\mathrm{sp}}\{u\} \text { for all } u \in \mathscr{L}^{1}(\mu)
$$

In what follows $H$ stands always for a (fixed) complex Hilbert space, $G$ stands for a fixed locally compact Abelian group and $\Gamma$ stands for the dual group of $G$. The group $\Gamma$ carries the usual locally compact Hausdorff topology. Furthermore, $\lambda$ and $\theta$ stand for a fixed pair of Haar measures on $G$ and $\Gamma$, respectively, satisfying the Plancherel relation. In what follows $\mathscr{L}^{p}(G)$ stands for $\mathscr{L}^{p}(\lambda), p=1,2$. For $u \in \mathscr{L}^{1}(G)$ we denote by $\mathscr{F} u$ the Fourier transform of $u$, i.e.,

$$
\mathscr{F} u(\gamma)=\int \overline{\gamma(x)} u(x) d \lambda(x), \quad \gamma \in \Gamma .
$$


By $\mathscr{F} u$ we denote even the Fourier transform of any function $u \in \mathscr{L}^{2}(G)$. For $u, v \in \mathscr{L}^{1}(G)$ the function $u * v$ is the convolution of $u$ and $v$. Finally, for any function $u: G \rightarrow C$ we put

$$
u_{a}(x)=u\left(a^{-1} x\right), \quad x \in G, \quad a \in G ; \quad u^{*}(x)=\overline{u\left(x^{-1}\right)}, \quad x \in G .
$$

The following definition is analogous to the definition of a second order stationary random measure or distribution; see e.g. Daley and Vere-Jones [8; p. 333] and Itô [13] or Gelfand and Vilenkin [9; pp. 262-263], respectively.

Definition 1.1. A vector measure $\mu: \mathscr{K}_{C}(G) \rightarrow H$ is stationary, if

$$
\left(\mu\left(f_{a}\right) \mid \mu\left(g_{a}\right)\right)=(\mu(f) \mid \mu(g)) \text { for all } f, g \in \mathscr{K}_{C}(G), \quad a \in G .
$$

The following lemma follows directly from the way to define the semi-variation $\mu^{\bullet}$ of a vector measure $\mu$ (cf. [18; pp. 65-66]).

Lemma 1.2. Let $\mu: \mathscr{K}_{C}(G) \rightarrow H$ be a stationary vector measure. Then

$$
\mu^{\cdot}\left(u_{a}\right)=\mu^{\cdot}(u) \text { for all } u: G \rightarrow R^{+} \cup\{+\infty\} \text { and } a \in G ;
$$

and $u \in \mathscr{L}^{1}(\mu)$ if and only if $u_{a} \in \mathscr{L}^{1}(\mu)$ for all $a \in G$.

Recall that a function $p: G \rightarrow C$ is called positive definite, if

$$
\sum_{j=1}^{m} \sum_{k=1}^{m} a_{j} \bar{a}_{k} p\left(x_{j}^{-1} x_{k}\right) \geqq 0
$$

for all $a_{j} \in C ; x_{j} \in G ; j=1, \ldots, m ; m \in N$.

Theorem 1.3. Let $\mu: \mathscr{K}_{C}(G) \rightarrow H$ be a stationary vector measure. Then for any $u \in \mathscr{L}^{1}(\mu)$ the function $F_{u}: G \rightarrow C$ defined by

$$
F_{u}(x)=\left(\int u d \mu \mid \int u_{x} d \mu\right), \quad x \in G,
$$

is a bounded continuous positive definite function; and there exists a uniquely determined bounded positive Radon measure $v_{u}: \mathscr{K}_{C}(\Gamma) \rightarrow C$ such that

$$
F_{u}(x)=\int \gamma(x) d v_{u}(\gamma), \quad x \in G,
$$

and

$$
\int\left(v * w^{*}\right)(x) \overline{F_{u}(x)} d \lambda(x)=\int \mathscr{\mathscr { F }} v \overline{\mathscr{F}} w d v_{u}
$$

for all $v, w \in \mathscr{L}^{1}(G)$.

Proof. Let $u \in \mathscr{L}^{1}(\mu)$ be given.

First we note that it follows from Lemma 1.2 and from the way to define the integral with respect to a vector measure (cf. [18; p. 69]) that

$$
\left(\int v_{a} d \mu \mid \int w_{a} d \mu\right)=\left(\int v d \mu \mid \int w d \mu\right)
$$

for all $v, w \in \mathscr{L}^{1}(\mu)$ and $a \in G$. The positive definiteness of the function $F_{u}$ follows then directly from (1.4). 
Clearly,

i.e., $F_{u}$ is bounded.

$$
\left|F_{u}(x)\right| \leqq\left\|\int u d \mu\right\|^{2}, \quad x \in G,
$$

In order to show that $F_{u}$ is continuous we first note that the continuity of any function of the type $F_{f}, f \in \mathscr{K}_{C}(G)$, follows from the continuity of $\mu$ and from the uniform continuity of the corresponding function $f$. The continuity of the function $F_{u}$ follows then in a straightforward way, by applying Lemma 1.2, from the way to define the integral with respect to a vector measure (cf. [18; p. 69]).

The existence of a uniquely determined bounded positive Radon measure $v_{u}: \mathscr{K}_{C}(\Gamma) \rightarrow C$ satisfying (1.2) follows now from the well-known Bochner representation theorem for continuous positive definite functions.

Finally, the formula (1.3) follows from (1.2) by a straightforward application of Fubini's theorem.

The theorem is proved.

Definition 1.4. Let $\mu: \mathscr{K}_{C}(G) \rightarrow H$ be a stationary vector measure and let $u \in \mathscr{L}^{1}(\mu)$. Then the uniquely determined bounded positive Radon measure $v_{u}$ on $\Gamma$ satisfying (1.2) is called the Bochner measure corresponding to the pair $(\mu, u)$.

Lemma 1.5. Let $\mu: \mathscr{K}_{C}(G) \rightarrow H$ be a vector measure. Then $f * u \in \mathscr{L}^{1}(\mu)$ for all $f \in \mathscr{K}_{C}(G), u \in \mathscr{L}^{1}(\mu) \cap \mathscr{L}^{1}(G)$.

Proof. Throughout the proof, by $\mu_{z}$ we denote the Radon measure defined by

$$
\left.\mu_{z}(f)=(\mu(f)) \mid z\right), \quad f \in \mathscr{K}_{C}(G) ; \quad z \in H .
$$

Recall that $\mu_{z}^{\cdot} \leqq\|z\| \mu^{\bullet}$ for all $z \in H$.

Since $\mu: \mathscr{K}_{C}(G) \rightarrow H$ is a vector measure with values in a Hilbert space, it is enough to show that $f * u \in \mathscr{L}^{1}\left(\mu_{z}\right)$ for all $z \in H$ (cf. [18; Théorème 5.6, Théorème 5.3 and Proposition 5.1]).

Suppose $z \in H, f \in \mathscr{K}_{C}(G)$ and $u \in \mathscr{L}^{1}(\mu) \cap \mathscr{L}^{1}(G) ; f \geqq 0, u \geqq 0$, are given. Since $f \in \mathscr{K}_{C}(G)$, the function $f * u$ is continuous. Let $K \subset G$ be a compact set and let $\chi_{K}$ be its characteristic function. Then $\chi_{K}(f * u) \in \mathscr{L}^{1}\left(\mu_{z}\right)$. Furthermore, by Fubini's theorem

$$
\begin{aligned}
\int \chi_{K}(f * u) d\left|\mu_{z}\right| & =\int \chi_{K}(x)\left(\int f(s) u\left(s^{-1} x\right) d \lambda(s)\right) d\left|\mu_{z}\right|(x) \\
& =\int f(s)\left(\int \chi_{K}(x) u\left(s^{-1} x\right) d \mu_{z} \mid(x)\right) d \lambda(s) \\
& \leqq\|z\| \mu \cdot(u) \int f d \lambda .
\end{aligned}
$$

It then follows that the function $f * u$ is $\mu_{z}$-integrable and, a fortiori, $\mu$-integrable.

The lemma is proved.

Lemma 1.6. Let $\mu: \mathscr{K}_{C}(G) \rightarrow H$ be a stationary vector measure. If $u \in \mathscr{L}^{1}(\mu) \cap$ $\mathscr{L}^{1}(G)$ and if $v_{u}$ is the Bochner measure corresponding to $(\mu, u)$, then

for all $f, g \in \mathscr{K}_{C}(G)$.

$$
\left(\int f * u d \mu \mid \int g * u d \mu\right)=\int \mathscr{F} g \overline{\mathscr{F} h} d v_{u}
$$


Proof. Suppose $u \in \mathscr{L}^{1}(\mu) \cap \mathscr{L}^{1}(G)$. Since the Bochner measure $v_{u}$ corresponding to $(\mu, u)$ is bounded, all the functions $\mathscr{\mathscr { F }} f \overline{\mathscr{F} g} ; f, g \in \mathscr{K}_{C}(G)$, are $v_{u}$-integrable. Furthermore, by Lemma 1.5, the functions $f * u$ and $g * u$ are $\mu$-integrable.

In the following lines we apply several times Fubini's theorem. Its use is justified, since the function

$$
F_{g * u, u}(x)=\left(\int g * u d \mu \mid \int u_{x} d \mu\right), \quad x \in G,
$$

is continuous and bounded and since $\mathscr{L}^{1}(\mu) \subset \mathscr{L}^{1}\left(\mu_{z}\right)$ for all $z \in H$; here $\mu_{z}$ is the Radon measure defined as in (1.5).

Denote

Then

$$
\begin{gathered}
\mu_{g * u}(h)=\left(\mu(h) \mid \int g * u d \mu\right), \quad h \in \mathscr{K}_{C}(G), \\
F_{u}(x)=\left(\int u d \mu \mid \int u_{x} d \mu\right), \quad x \in G .
\end{gathered}
$$

$$
\begin{aligned}
\left(\int f * u d \mu \mid \int g * u d \mu\right) & =\int f * u d \mu_{g * u} \\
& =\int\left(\int u\left(x^{-1} s\right) f(x) d \lambda(x)\right) d \mu_{g * u}(s) \\
& =\int f(x)\left(\int u\left(x^{-1} s\right) d \mu_{g * u}(s)\right) d \lambda(x) \\
& =\int f(x)\left(\int u_{x} d \mu \mid \int g * u d \mu\right) d \lambda(x) .
\end{aligned}
$$

In a similar way we get

$$
\begin{aligned}
\left(\int g * u d \mu \mid \int u_{x} d \mu\right) & =\int g(y)\left(\int u_{y} d \mu \mid \int u_{x} d \mu\right) d \lambda(y) \\
& =\int g(y) F_{u}\left(y^{-1} x\right) d \lambda(y) .
\end{aligned}
$$

Thus, by applying Fubini's theorem and (1.2) we get

$$
\begin{aligned}
\left(\int f * u d \mu \mid \int g * u d \mu\right) & =\int f(x) \overline{\left(\int g(y) F_{u}\left(y^{-1} x\right) d \lambda(y)\right)} d \lambda(x) \\
& =\int \mathscr{F} f \overline{\mathscr{F} g} d v_{u} .
\end{aligned}
$$

The lemma is proved.

Lemma 1.7. Let $\mu: \mathscr{K}_{C}(G) \rightarrow H$ be a stationary vector measure. Suppose $u \in \mathscr{L}^{1}(\mu) \cap \mathscr{L}^{1}(G)$. Then for all $f \in \mathscr{K}_{C}(G)$

$$
v_{f * u}=|\mathscr{F} f|^{2} \cdot v_{u},
$$

where $v_{f * u}$ and $v_{u}$ are the Bochner measures corresponding to $(\mu, f * u)$ and $(\mu, u)$, respectively.

Proof. Let $u \in \mathscr{L}^{1}(\mu) \cap \mathscr{L}^{1}(G)$ and $f \in \mathscr{K}_{C}(G)$. Then, by Lemma 1.6,

for all $h \in \mathscr{K}_{C}(G)$.

$$
\begin{aligned}
\int|\mathscr{F} h|^{2}|\mathscr{F} f|^{2} d v_{u} & =\left(\int h * f * u d \mu \mid \int h * f * u d \mu\right) \\
& =\int|\mathscr{F} h|^{2} d v_{f * u}
\end{aligned}
$$


The assertion then follows in a straightforward way from the boundedness of the positive Radon measures $v_{u}: \mathscr{K}_{C}(\Gamma) \rightarrow C$ and $v_{f * u}: \mathscr{K}_{C}(\Gamma) \rightarrow C$ and from the obvious fact that any function $\varphi \in \mathscr{K}_{C}(\Gamma)$ can be approximated (in the supremum norm topology) by the elements in the linear span of the functions $|\mathscr{F} h|^{2}, h \in \mathscr{K}_{C}(G)$.

The lemma is proved.

2. Positive definite translation invariant bimeasures. In this section we present a Bochner representation theorem for positive definite translation invariant bimeasures.

Recall that a continuous bilinear mapping $B: \mathscr{K}_{C}(G) \times \mathscr{K}_{C}(G) \rightarrow C$ is called a bimeasure on $G \times G$.

Definition 2.1. A bimeasure $B: \mathscr{K}_{C}(G) \times \mathscr{K}_{C}(G) \rightarrow C$ is called positive definite, if

$$
B(f, \bar{f}) \geqq 0 \text { for all } f \in \mathscr{K}_{C}(G)
$$

it is called translation invariant, if

$$
B\left(f_{a}, g_{a}\right)=B(f, g) \text { for all } f, g \in \mathscr{K}_{C}(G) \text { and } a \in G .
$$

Theorem 2.2. A bimeasure $B: \mathscr{K}_{C}(G) \times \mathscr{K}_{C}(G) \rightarrow C$ is positive definite and translation invariant, if and only if there exist a Hilbert space $M$ and a stationary vector measure $\mu: \mathscr{K}_{C}(G) \rightarrow M$ such that

$$
(\mu(f) \mid \mu(g))=B(f, \bar{g}) \text { for all } f, g \in \mathscr{K}_{C}(G) .
$$

Proof. Let $M$ be a Hilbert space and let $\mu: \mathscr{K}_{C}(G) \rightarrow M$ be a stationary vector measure. It is clear that the bilinear mapping $B: \mathscr{K}_{C}(G) \times \mathscr{K}_{C}(G) \rightarrow C$ defined by

$$
B(f, g)=(\mu(f) \mid \mu(\bar{g})), \quad f, g \in \mathscr{K}_{C}(G),
$$

is a positive definite translation invariant bimeasure on $G \times G$.

On the other hand, suppose $B: \mathscr{K}_{C}(G) \times \mathscr{K}_{C}(G) \rightarrow C$ is a positive definite translation invariant bimeasure on $G \times G$. Consider the positive definite kernel $Q: \mathscr{K}_{C}(G) \times \mathscr{K}_{C}(G) \rightarrow C$,

$$
Q(f, g)=\overline{B(f, \bar{g}}), \quad f, g \in \mathscr{K}_{C}(G),
$$

and the reproducing kernel Hilbert space $H(Q)$ defined by $Q$ (cf. Aronszajn [2; p. 344]).

For $g \in \mathscr{K}_{C}(G)$ define $Q_{g}: \mathscr{K}_{C}(G) \rightarrow C$ by

$$
Q_{g}(f)=Q(f, g), \quad f, g \in \mathscr{K}_{C}(G) .
$$

Then the mapping $\mu: \mathscr{K}_{C}(G) \rightarrow H(Q)$,

$$
\mu(f)=Q_{f}, \quad f \in \mathscr{K}_{C}(G),
$$


is a well-defined linear mapping. Furthermore, since $B: \mathscr{K}_{C}(G) \times \mathscr{K}_{C}(G) \rightarrow C$ is a continuous bilinear mapping, it follows that $\mu: \mathscr{K}_{C}(G) \rightarrow H(Q)$ is a continuous linear mapping, i.e., $\mu$ is a vector measure. Finally,

$$
(\mu(f) \mid \mu(g))=\left(Q_{f}, Q_{g}\right)=\overline{Q(f, g)}=B(f, \tilde{g}), \quad f, g \in \mathscr{K}_{C}(G) ;
$$

which proves the theorem.

Definition 2.3. Let $M$ be a Hilbert space and let $\mu: \mathscr{K}_{C}(G) \rightarrow M$ be a vector measure. The bimeasure $B: \mathscr{K}_{C}(G) \times \mathscr{K}_{C}(G) \rightarrow C$ defined by

$$
B(f, g)=(\mu(f) \mid \mu(\bar{g})), \quad f, g \in \mathscr{K}_{C}(G),
$$

is called the bimeasure defined by $\mu$.

Let $B: \mathscr{K}_{C}(G) \times \mathscr{K}_{C}(G) \rightarrow C$ be a bimeasure and let $(u, v)$ be a $B$-integrable pair of functions $u: G \rightarrow C, v: G \rightarrow C$. Then by

$$
\int(u, v) d B
$$

we denote the integral of $(u, v)$ with respect to $B$. (For the definition of the integral with respect to a bimeasure we refer to [18; pp. 144-145] and to the references given there.)

The following theorem was proved in [15; Theorem 2.4.11].

Theorem 2.4. Let $M$ be a Hilbert space and let $\mu: \mathscr{K}_{C}(G) \rightarrow M$ be a vector measure. If $B: \mathscr{K}_{C}(G) \times \mathscr{K}_{C}(G) \rightarrow C$ is the bimeasure defined by $\mu$, then all the pairs $(u, \bar{v}) ; u, v \in \mathscr{L}^{1}(\mu)$, are B-integrable and

$$
\left(\int u d \mu \mid \int v d \mu\right)=\int(u, \bar{v}) d B .
$$

We are now ready to prove the Bochner representation theorem for positive definite translation invariant bimeasures.

Theorem 2.5. Let $B: \mathscr{K}_{C}(G) \times \mathscr{K}_{C}(G) \rightarrow C$ be a mapping. Then $B$ is a positive definite translation invariant bimeasure, if and only if there exists a uniquely determined positive Radon measure $v$ on $\Gamma$ such that $\mathscr{\mathscr { F }} f \overline{\mathscr{F} g} \in \mathscr{L}^{1}(v)$ and

$$
B(f, \bar{g})=\int \mathscr{F} f \overline{\mathscr{F} g} d v \text { for all } f, g \in \mathscr{K}_{C}(G) .
$$

If $B: \mathscr{K}_{C}(G) \times \mathscr{K}_{C}(G) \rightarrow C$ is a positive definite translation invariant bimeasure, if $v$ is the positive Radon measure on $\Gamma$ satisfying (2.1) and if $\mu: \mathscr{K}_{C}(G) \rightarrow M$ is any stationary vector measure with values in a Hilbert space $M$ such that $B$ is the bimeasure defined by $\mu$, then for all $u, v \in \mathscr{L}^{1}(\mu) \cap \mathscr{L}^{1}(G)$ the pair $(u, \bar{v})$ is B-integrable, $\mathscr{F} u \overline{\mathscr{F}} v \in \mathscr{L}^{1}(v)$ and

$$
\int(u, \bar{v}) d B=\int \mathscr{F} u \overline{\mathscr{F} v} d v .
$$

Especially, (2.2) is valid for all bounded Borel functions $u: G \rightarrow C, v: G \rightarrow C$ with compact support. 
Proof. Suppose $B: \mathscr{K}_{C}(G) \times \mathscr{K}_{C}(G) \rightarrow C$ is a positive definite translation invariant bimeasure and suppose $\mu: \mathscr{K}_{C}(G) \rightarrow M$ is a stationary vector measure with values in a Hilbert space $M$ such that $B$ is the bimeasure defined by $\mu$ (cf. Theorem 2.2).

We start by constructing a positive Radon measure $v$ on $\Gamma$. The construction is fairly standard (cf. Rudin [17; p. 22], Berg [4], [5], Berg and Forst [6; pp. 19-20] and Argabright and Gil de Lamadrid [1; p. 14]).

Throughout the proof $v_{w}$ stands for the Bochner measure corresponding to the pair $(\mu, w)$, where $w \in \mathscr{L}^{1}(\mu) \cap \mathscr{L}^{1}(G)$.

Let $K \subset \Gamma$ be a compact set. Fix a number $d>0$ and choose a function $h \in \mathscr{K}_{C}(G)$ such that

Define

$$
|\mathscr{F} h(\gamma)|^{2}>d, \quad \gamma \in K
$$

$$
A_{h}(\varphi)=\int \varphi \frac{1}{|\mathscr{F} h|^{2}} d v_{h}, \quad \varphi \in \mathscr{K}_{C}(\Gamma ; K) .
$$

Then $A_{h}: \mathscr{K}_{C}(\Gamma ; K) \rightarrow C$ is a well-defined linear mapping. Furthermore,

$$
\begin{aligned}
\left|A_{h}(\varphi)\right| & \leqq \sup |\varphi| \sup _{\gamma \in K} \frac{1}{|\mathscr{F} h(\gamma)|^{2}} \int d v_{h} \\
& \leqq \sup |\varphi| d^{-1} \int d v_{h}, \quad \varphi \in \mathscr{K}_{C}(\Gamma ; K) ;
\end{aligned}
$$

which proves that the linear mapping $A_{h}: \mathscr{K}_{C}(\Gamma ; K) \rightarrow C$ is continuous, if the space $\mathscr{K}_{C}(\Gamma ; K)$ carries the topology defined by the supremum norm.

Suppose now $K^{\prime} \subset \Gamma$ is any compact set such that $K \subset K^{\prime}$ and suppose $h^{\prime} \in \mathscr{K}_{C}(G)$ is a function such that $\left|\mathscr{F} h^{\prime}(\gamma)\right|^{2}>d^{\prime}, \gamma \in K^{\prime}$; for some constant $d^{\prime}>0$. Then, by Lemma 1.7,

$$
\begin{aligned}
A_{h}(\varphi) & =\int \varphi \frac{1}{|\mathscr{F} h|^{2}} d v_{h}=\int \varphi \frac{1}{|\widetilde{F} h|^{2} \mid \mathscr{F} h^{\prime 2}} d v_{h * h^{\prime}} \\
& =\int \varphi \frac{1}{\left|\mathscr{F} h^{\prime}\right|^{2}} d v_{h^{\prime}}=A_{h^{\prime}}(\varphi) \text { for all } \varphi \in \mathscr{K}_{C}(\Gamma: K) .
\end{aligned}
$$

Thus, the mapping

$$
v(\varphi)=\int \varphi \frac{1}{\left|\mathscr{F} h_{(\varphi)}\right|^{2}} d v_{h_{(\varphi)}}, \quad \varphi \in \mathscr{K}_{C}(\Gamma)
$$

where $h_{(\varphi)} \in \mathscr{K}_{C}(G)$ is such a function that for some constant $d^{\prime \prime}>0$

$$
\left|\mathscr{F} h_{(\varphi)}(\gamma)\right|^{2}>d^{\prime \prime} \quad \text { for all } \gamma \in \operatorname{supp} \varphi,
$$

is a well-defined linear mapping. Furthermore, it follows from (2.3) that the linear mapping $v: \mathscr{K}_{C}(\Gamma) \rightarrow C$ is continuous, i.e., $v$ is a Radon measure on $\Gamma$. The positivity of all the Radon measures $v_{h}, h \in \mathscr{K}_{C}(G)$, implies that even $v$ is positive.

Next we show that $v$ satisfies (2.1).

Suppose $u, v \in \mathscr{L}^{1}(\mu) \cap \mathscr{L}^{1}(G)$. Then, by Theorem 2.4 the pair $(u, \bar{v})$ is $B$-integrable. 
Let $\varphi \in \mathscr{K}_{C}(\Gamma)$ be given. Choose a function $h \in \mathscr{K}_{C}(G)$ such that for some constant $d>0$ one has $|\mathscr{F} h(\gamma)|^{2}>d$ for all $\gamma \in \operatorname{supp} \varphi$. By the definition of $v$ and by Lemma 1.7 we then get

$$
\begin{aligned}
\int \varphi d v_{u} & =\int \frac{\varphi}{|\mathscr{F} h|^{2}} d v_{h * u} \\
& =\int \varphi|\mathscr{F} u|^{2} \frac{1}{|\mathscr{F} h|^{2}} d v_{h}=\int \varphi|\mathscr{F} u|^{2} d v ;
\end{aligned}
$$

which proves that $|\mathscr{F} u|^{2} \cdot v=v_{u}$. Since $v_{u}$ is a bounded Radon measure, it then follows that

$$
\int|\mathscr{F} u|^{2} d v=\int d v_{u}
$$

Thus, by Theorem 2.4 and Theorem 1.3,

$$
\int(u, \bar{u}) d B=\left\|\int u d \mu\right\|^{2}=\int d v_{u}=\int|\mathscr{F} u|^{2} d v .
$$

Furthermore, by applying a well-known polarization formula, we then get

$$
\int(u, \bar{v}) d B=\int \mathscr{F} u \overline{\mathscr{F} v} d v \text { for all } u, v \in \mathscr{L}^{1}(\mu) \cap \mathscr{L}^{1}(G),
$$

Especially, the representation (2.5) is valid for all bounded Borel functions $u: G \rightarrow C$, $v: G \rightarrow C$ with compact support, since all these functions are integrable with respect to any vector measure with values in a Hilbert space (cf. [18; p. 101]).

We still prove that the positive Radon measure $v$ on $\Gamma$ satisfying (2.1) is uniquely determined.

Suppose there exist two positive Radon measures $v$ and $v^{\prime}$ on $\Gamma$ satisfying

$$
B(f, \bar{g})=\int \mathscr{\mathscr { F }} f \overline{\mathscr{F} g} d v=\int \mathscr{\mathscr { F }} f \overline{\mathscr{F} g} d v^{\prime} \quad \text { for all } f, g \in \mathscr{K}_{C}(G) .
$$

By applying exactly the same arguments as in the proof of Lemma 1.7 we then get

$$
|\mathscr{F} h|^{2} \cdot v=|\mathscr{F} h|^{2} \cdot v^{\prime} \quad \text { for all } h \in \mathscr{K}_{C}(G) \text {. }
$$

Furthermore, by applying the same technique as in proving (2.4) we then get that $v$ and $v^{\prime}$ must be identical.

In order to complete the proof we must still show that any mapping $B: \mathscr{K}_{C}(G) \times$ $\mathscr{K}_{C}(G) \rightarrow C$ defined by (2.1), when $v$ is given, is a positive definite translation invariant bimeasure.

Thus, let $v: \mathscr{K}_{C}(\Gamma) \rightarrow C$ be a positive Radon measure such that $\mathscr{\mathscr { F }} f \overline{\mathscr{F} g} \in \mathscr{L}^{1}(v)$ for all $f, g \in \mathscr{K}_{C}(G)$. It is clear that the mapping $B: \mathscr{K}_{C}(G) \times \mathscr{K}_{C}(G) \rightarrow C$ defined by

$$
B(f, \bar{g})=\int \mathscr{F} f \overline{\mathscr{F} g} d v, \quad f, g \in \mathscr{K}_{C}(G),
$$

is a well-defined bilinear mapping satisfying the conditions

$$
B(f, \bar{f}) \geqq 0, \quad B\left(f_{a}, g_{a}\right)=B(f, g) \text { for all } f, g \in \mathscr{K}_{C}(G) ; a \in G .
$$

It follows from the way to define the locally convex inductive limit topology of $\mathscr{K}_{C}(G)$ that in order to show the continuity of $B$ it is enough to show that $B$ is separately continuous (cf. [18; p. 144]). 
Let $g \in \mathscr{K}_{C}(G)$ be fixed. We show that the linear mapping $B(., \bar{g}): \mathscr{K}_{C}(G) \rightarrow C$, $B(., \bar{g})(f)=B(f, \bar{g}), f \in \mathscr{K}_{C}(G)$, is continuous. Since $g \in \mathscr{K}_{C}(G)$, the support of $\mathscr{F} g$ is $\sigma$-compact (cf. Benedetto [3; p.20]) and, a fortiori, even the support of the Radon measure $\mathscr{F} g . v$ is $\sigma$-compact. Let $L_{n} \subset \Gamma, n \in N$, be an increasing family of compact sets such that

$$
\operatorname{supp}(\mathscr{F} g . v) \subset \bigcup_{n \in N} L_{n} .
$$

Let $K \subset G$ be a compact set. For $n \in N$ define $B_{n}(., \bar{g}): \mathscr{K}_{C}(G ; K) \rightarrow C$ by

$$
B_{n}(., \bar{g})(f)=\int_{L_{n}} \mathscr{F} f \overline{\mathscr{F} g} d v, \quad f \in \mathscr{K}_{C}(G ; K) .
$$

Then $B_{n}(., \bar{g})$ is clearly a well-defined linear mapping. Furthermore,

$$
\left|B_{n}(., \bar{g})(f)\right| \leqq \sup |f| \int_{K} d \lambda \int_{L_{n}}|\mathscr{F} g| d v, \quad f \in \mathscr{K}_{C}(G ; K),
$$

which shows that the linear mapping $B_{n}(., \bar{g}): \mathscr{K}_{C}(G ; K) \rightarrow C$ is continuous. It follows from the Lebesgue convergence theorem that

$$
\begin{aligned}
\lim _{n \rightarrow \infty} B_{n}(., \bar{g})(f) & =\lim _{n \rightarrow \infty} \int_{L_{n}} \mathscr{\mathscr { F } f} \overline{\mathscr{F} g} d v \\
& =\int \mathscr{\mathscr { F }} f \overline{\mathscr{F} g} d v=B(., \bar{g})(f), \quad f \in \mathscr{K}_{C}(G ; K) .
\end{aligned}
$$

Thus, the restriction of $B(., \bar{g})$ to the Banach space $\mathscr{K}_{C}(G ; K)$ is a pointwise limit of the continuous linear mappings $B_{n}(., \bar{g}): \mathscr{K}_{C}(G ; K) \rightarrow C, n \in N$. Therefore, it follows from the uniform boundedness principle that even the linear mapping $B(., \bar{g})(f)=B(f, \bar{g}), f \in \mathscr{K}_{C}(G ; K)$, is continuous (cf. Horváth [12; pp. 62-63]). It then follows that even the mapping $B(., \bar{g}): \mathscr{K}_{C}(G) \rightarrow C$ is continuous.

The separate continuity of $B: \mathscr{K}_{C}(G) \times \mathscr{K}_{C}(G) \rightarrow C$ follows then by symmetry.

The theorem is proved.

Example 2.6. Recall that a Radon measure $\mu: \mathscr{K}_{C}(G) \rightarrow C$ is called positive definite, if

$$
\int f * f^{*} d \mu \geqq 0 \text { for all } f \in \mathscr{K}_{C}(G)
$$

(cf. [4], [5], [6; p. 18] or [1; p. 23]).

Let $\mu: \mathscr{K}_{C}(G) \rightarrow C$ be a positive definite Radon measure. Define a mapping $B: \mathscr{K}_{C}(G) \times \mathscr{K}_{C}(G) \rightarrow C$ by

$$
B(f, \bar{g})=\int f * g^{*} d \mu, \quad f, g \in \mathscr{K}_{C}(G) .
$$

Then $B$ is a well-defined bilinear mapping. Furthermore, it follows from the Bochner representation theorem for positive definite Radon measures (cf. [4], [5], [6; p. 19] or $[1 ;$ p. 23]) that there exists a uniquely determined positive Radon measure $v: \mathscr{K}_{C}(\Gamma) \rightarrow C$ such that for all $f, g \in \mathscr{K}_{C}(G)$ one has $\mathscr{\mathscr { F }} f \overline{\mathscr{F} g} \in \mathscr{L}^{1}(v)$ and

$$
B(f, \bar{g})=\int f * g^{*} d \mu=\int \mathscr{F} f \overline{\mathscr{F} g} d v .
$$


Thus, it follows from Theorem 2.5 that $B$ is a positive definite translation invariant bimeasure.

We show that there exist positive definite translation invariant bimeasures $B: \mathscr{K}_{C}(G) \times \mathscr{K}_{C}(G) \rightarrow C$ for which there does not exist any positive definite Radon measure $\mu: \mathscr{K}_{C}(G) \rightarrow C$ satisfying (2.6).

Choose $G=R$. Suppose $h: R \rightarrow R$ is a non-negative bounded Lebesgue measurable function. Then the positive Radon measure

$$
v=h \cdot d x
$$

has the property that $|\mathscr{F} f|^{2} \in \mathscr{L}^{1}(v)$ for all $f \in \mathscr{K}_{C}(R)$, i.e., $v$ is the Fourier transform of a positive definite translation invariant bimeasure (cf. Theorem 2.5). If all positive Radon measures $v: \mathscr{K}_{C}(R) \rightarrow C$ of the type (2.7) were Fourier transforms of positive definite Radon measures, then even the function

$$
h(x)=\operatorname{sign} x, \quad x \in R,
$$

were the Fourier transform of a Radon measure on $R$. But it is well-known that the function $i \pi h$ is the Fourier transform of the so-called Hilbert distribution (which is not a Radon measure on $R$ ).

Next we consider some special cases of Theorem 2.5.

Theorem 2.7. Let $B: \mathscr{K}_{C}(G) \times \mathscr{K}_{C}(G) \rightarrow C$ be a positive definite translation invariant bimeasure and let $\mu: \mathscr{K}_{C}(G) \rightarrow M$ be any stationary vector measure with values in a Hilbert space $M$ such that $B$ is the bimeasure defined by $\mu$. Then the uniquely determined positive Radon measure $v: \mathscr{K}_{C}(\Gamma) \rightarrow C$ satisfying $(2.1)$ is bounded, if and only if there exists a constant $c>0$ such that

$$
B(f, \bar{f}) \leqq c \sup |\mathscr{F} f|^{2} \text { for all } f \in \mathscr{K}_{C}(G) .
$$

If (2.8) is satisfied, then $\mathscr{L}^{1}(G) \subset \mathscr{L}^{1}(\mu)$; and for any $u, v \in \mathscr{L}^{1}(G)$ the pair $(u, \bar{v})$ is $B$-integrable and

$$
\int(u, \bar{v}) d B=\int \mathscr{F} u \overline{\mathscr{F} v} d v
$$

Proof. The condition (2.8) is clearly satisfied in the case when $v$ is bounded. On the other hand, let $B: \mathscr{K}_{C}(G) \times \mathscr{K}_{C}(G) \rightarrow C$ be a positive definite translation invariant bimeasure satisfying (2.8) and let $\mu: \mathscr{K}_{C}(G) \rightarrow M$ be a stationary vector measure such that $B$ is the bimeasure defined by $\mu$ (cf. Theorem 2.2). It follows from the condition (2.8) that

$$
\|\mu(f)\| \leqq c^{1 / 2} \int|f| d \lambda \quad \text { for all } f \in \mathscr{K}_{C}(G) .
$$

Thus, $\mathscr{L}^{1}(G) \subset \mathscr{L}^{1}(\mu)$ (cf. [18; p. 69]). By Theorem 2.4 we then get that for any $u, v \in \mathscr{L}^{1}(G)$ the pair $(u, \bar{v})$ is $B$-integrable. Furthermore, by Theorem 2.5 ,

$$
\int(u, \bar{v}) d B=\int \mathscr{F} u \overline{\mathscr{F} v} d v,
$$

where $v: \mathscr{K}_{C}(\Gamma) \rightarrow C$ is the positive Radon measure satisfying (2.1).

We still show that $v$ is bounded. 
Let $K \subset \Gamma$ be a compact set. Choose a function $h \in \mathscr{K}_{C}(G)$ such that for some constant $1>d>0$ one has

$$
|\mathscr{F} h(\gamma)|^{2}>d, \quad \gamma \in K ; \quad|\mathscr{F} h(\gamma)|^{2}<2, \quad \gamma \in \Gamma .
$$

It then follows from (2.8) that for the Bochner measure $v_{h}$ corresponding to $(\mu, h)$ we have

Thus,

$$
\int d v_{h}=B(h, \bar{h}) \leqq c \sup |\mathscr{F} h|^{2}<2 c .
$$

$$
\begin{aligned}
\left|\int \varphi d v\right| & =\left|\int \varphi \frac{1}{|\mathscr{F} h|^{2}} d v_{h}\right| \\
& \leqq \sup |\varphi| d^{-1} \int d v_{h} \leqq 2 c d^{-1} \sup |\varphi|
\end{aligned}
$$

for all $\varphi \in \mathscr{K}_{C}(\Gamma ; K)$. Since the constant $2 c d^{-1}$ is independent of the set $K$, it then follows that the Radon measure $v$ is bounded.

The theorem is proved.

Theorem 2.8. Let $B: \mathscr{K}_{C}(G) \times \mathscr{K}_{C}(G) \rightarrow C$ be a positive definite translation invariant bimeasure, let $v: \mathscr{K}_{C}(\Gamma) \rightarrow C$ be the uniquely determined positive Radon measure satisfying (2.1) and let $\mu: \mathscr{K}_{C}(G) \rightarrow M$ be any stationary vector measure with values in a Hilbert space $M$ such that $B$ is the bimeasure defined by $\mu$. Suppose there exist constants $c>0, c^{\prime}>0$ such that

$$
\begin{aligned}
& B(f, \bar{f}) \leqq c \int|f|^{2} d \lambda \quad \text { for all } f \in \mathscr{K}_{C}(G), \\
& v\left(|\varphi|^{2}\right) \leqq c^{\prime} \int|\varphi|^{2} d \theta \quad \text { for all } \varphi \in \mathscr{K}_{C}(\Gamma),
\end{aligned}
$$

then $\mathscr{L}^{2}(G) \subset \mathscr{L}^{1}(\mu), \mathscr{L}^{2}(\Gamma) \subset \mathscr{L}^{2}(v)$ and for any $u, v \in \mathscr{L}^{2}(G)$ the pair $(u, \bar{v})$ is $B$-integrable and

$$
\int(u, \bar{v}) d B=\int \mathscr{F} u \overline{\mathscr{F} v} d v .
$$

Proof. If (2.9) holds, then $\mathscr{L}^{2}(G) \subset \mathscr{L}^{1}(\mu)$ and

$$
\left\|\int h d \mu\right\|^{2} \leqq c \int|h|^{2} d \lambda \text { for all } h \in \mathscr{L}^{2}(G)
$$

(cf. [18; p. 69]). Similarly, the condition $\left(2.9^{\prime}\right)$ implies that $\mathscr{L}^{2}(\Gamma) \subset \mathscr{L}^{2}(v)$ and

$$
\int|h|^{2} d v \leqq c^{\prime} \int|h|^{2} d \theta \quad \text { for all } \quad h \in \mathscr{L}^{2}(\Gamma)
$$

Since $\mathscr{L}^{2}(G) \subset \mathscr{L}^{1}(\mu)$, it follows from Theorem 2.4 that for any $u, v \in \mathscr{L}^{2}(G)$ the pair $(u, \bar{v})$ is $B$-integrable and

$$
\int(u, \bar{v}) d B=\left(\int u d \mu \mid \int v d \mu\right) .
$$

Furthermore, a straightforward application of the inequalities (2.11), (2.11') and the well-known polarization formula shows that even (2.10) holds.

The theorem is proved. 
3. A representation theorem for stationary vector measures. In this section we present a representation theorem for stationary vector measures with values in a Hilbert space. Our result is closely related to a representation theorem for second order stationary random distributions (cf. Itô [13] or Gelfand and Vilenkin [9; p. 271]).

First we recall some basic properties of orthogonally scattered vector measures with values in a Hilbert space.

Let $M$ be a Hilbert space and let $S$ be a locally compact Hausdorff space. Recall that a vector measure $\mu: \mathscr{K}_{C}(S) \rightarrow M$ is called orthogonally scattered, if

$$
(\mu(f) \mid \mu(g))=0 \text { for all } f, g \in \mathscr{K}_{C}(S) \text { such that } \operatorname{supp} f \cap \operatorname{supp} g=\emptyset .
$$

A vector measure $\mu: \mathscr{K}_{C}(S) \rightarrow M$ is orthogonally scattered, if and only if there exists a (uniquely determined) positive Radon measure $v$ on $S$ such that

$$
(\mu(f) \mid \mu(g))=v(f \bar{g}) \text { for all } f, g \in \mathscr{K}_{C}(S) .
$$

If (3.1) is valid, then $\mathscr{L}^{2}(v)=\mathscr{L}^{1}(\mu)$ and

$$
\left(\int u d \mu \mid \int v d \mu\right)=\int u \bar{v} d v \text { for all } u, v \in \mathscr{L}^{1}(\mu)
$$

(cf. Masani [14; Theorem 5.9] or [16; Theorem 24]).

Let $v: \mathscr{K}_{C}(S) \rightarrow C$ be a positive Radon measure on $S$. In what follows we consider the space $L^{2}(v)$, formed by all equivalence classes [ $u$ ] of locally $v$-almost everywhere equal functions $u: S \rightarrow C$ such that $|u|^{2}$ is $v$-integrable, as a Hilbert space with the inner product

$$
([u] \mid[v])=\int u \bar{v} d v, \quad[u],[v] \in L^{2}(v) .
$$

We shall use also the notation $[u]=u,[u] \in L^{2}(v)$.

The following lemma can be proved in a straightforward way by applying Theorem 24 in [16].

Lemma 3.1. Let $S$ be a locally compact Hausdorff space and let $v: \mathscr{K}_{C}(S) \rightarrow C$ be a positive Radon measure. Then the mapping $\mu: \mathscr{K}_{C}(S) \rightarrow L^{2}(v)$,

$$
\mu(f)=[f], \quad f \in \mathscr{K}_{C}(S),
$$

is an orthogonally scattered vector measure such that $\mathscr{L}^{1}(\mu)=\mathscr{L}^{2}(v), \overline{\mathrm{sp}}\{\mu\}=L^{2}(v)$,

and

$$
\int u d \mu=[u] \text { for all } u \in \mathscr{L}^{1}(\mu)
$$

$$
\left(\int u d \mu \mid \int v d \mu\right)=\int u \bar{v} d v \text { for all } u, v \in \mathscr{L}^{1}(\mu) \text {. }
$$

Lemma 3.2. Let $v: \mathscr{K}_{C}(\Gamma) \rightarrow C$ be a positive Radon measure. Suppose all the functions $|\mathscr{F} f|^{2}, f \in \mathscr{K}_{C}(G)$, are v-integrable. Then the set $\left\{\mathscr{F} f \mid f \in \mathscr{K}_{C}(G)\right\}$ is a dense linear subspace in $L^{2}(v)$. 
Proof. Since the set $\mathscr{K}_{C}(\Gamma)$ is a dense linear subspace in $L^{2}(v)$, it is enough to show that for a given $\varphi \in \mathscr{K}_{C}(\Gamma)$ and a given $\varepsilon>0$ there exists a function $f \in \mathscr{K}_{C}(G)$ such that

for some constant $c>0$.

$$
\left(\int|\mathscr{F} f-\varphi|^{2} d v\right)^{1 / 2}<c \varepsilon
$$

Choose functions $g, h \in \mathscr{K}_{C}(G)$ such that

and

$$
D(g, \varphi)=\sup _{\gamma \in \operatorname{supp} \varphi}|1-\mathscr{F} g(\gamma)|<\varepsilon
$$

$$
\sup |\varphi-\mathscr{F} h|<\varepsilon\left(\left(\int|\mathscr{F} g|^{2} d v\right)^{1 / 2}+1\right)^{-1} .
$$

Furthermore, $f=h * g \in \mathscr{K}_{C}(G)$ and

$$
\begin{aligned}
\left(\int|\varphi-\mathscr{F} h \mathscr{F} g|^{2} d v\right)^{1 / 2} & \leqq\left(\int|1-\mathscr{F} g|^{2}|\varphi|^{2} d v\right)^{1 / 2}+\left(\int|\varphi-\mathscr{F} h|^{2}|\mathscr{F} g|^{2} d v\right)^{1 / 2} \\
& \leqq D(g, \varphi)\left(\int|\varphi|^{2} d v\right)^{1 / 2}+\sup |\varphi-\mathscr{F} h|\left(\int|\mathscr{F} g|^{2} d v\right)^{1 / 2} \\
& <\varepsilon\left(\int|\varphi|^{2} d v\right)^{1 / 2}+\varepsilon .
\end{aligned}
$$

The lemma is proved.

We are now ready to present the representation theorem for stationary vector measures.

Theorem 3.3. A mapping $\mu: \mathscr{K}_{C}(G) \rightarrow H$ is a stationary vector measure, if and only if there exists a uniquely determined orthogonally scattered vector measure $\mu_{0}: \mathscr{K}_{C}(\Gamma) \rightarrow H$ such that $\mathscr{F} f \in \mathscr{L}^{1}\left(\mu_{0}\right)$ and

$$
\mu(f)=\int \mathscr{F} f d \mu_{0} \quad \text { for all } f \in \mathscr{K}_{C}(G) .
$$

Suppose $\mu: \mathscr{K}_{C}(G) \rightarrow H$ is a stationary vector measure and suppose $\mu_{0}: \mathscr{K}_{C}(\Gamma) \rightarrow H$ is the orthogonally scattered vector measure for which (3.2) is satisfied. Then $\overline{\operatorname{sp}}\left\{\mu_{0}\right\}=$ $\overline{\mathrm{sp}}\{\mu\} ; u \in \mathscr{L}^{1}\left(\mu_{0}\right)$ and

$$
\int u d \mu=\int \mathscr{F} u d \mu_{0} \quad \text { for all } \quad u \in \mathscr{L}^{1}(\mu) \cap \mathscr{L}^{1}(G) ;
$$

especially for all bounded Borel functions $u: G \rightarrow C$ with compact support. Suppose $B: \mathscr{K}_{C}(G) \times \mathscr{K}_{C}(G) \rightarrow C$ is the (positive definite translation invariant) bimeasure defined by $\mu$. If $v: \mathscr{K}_{C}(\Gamma) \rightarrow C$ is the uniquely determined positive Radon measure satisfying

then

$$
B(f, \bar{g})=\int \mathscr{F} f \overline{\mathscr{F}} g d v \text { for all } f, g \in \mathscr{K}_{C}(G),
$$

$$
\left(\int u d \mu_{0} \mid \int v d \mu_{0}\right)=\int u \bar{v} d v \text { for all } u, v \in \mathscr{L}^{2}(v) ;
$$

especially for all bounded Borel functions $u: \Gamma \rightarrow C, v: \Gamma \rightarrow C$ with compact support.

Proof. Suppose a mapping $\mu: \mathscr{K}_{C}(G) \rightarrow H$ can be represented in the form

$$
\mu(f)=\int \mathscr{F} f d \mu_{0}, \quad f \in \mathscr{K}_{C}(G),
$$


where $\mu_{0}: \mathscr{K}_{C}(\Gamma) \rightarrow H$ is an orthogonally scattered vector measure such that $\mathscr{F} f \in \mathscr{L}^{1}\left(\mu_{0}\right)$ for all $f \in \mathscr{K}_{C}(G)$. Let $v: \mathscr{K}_{C}(\Gamma) \rightarrow C$ be the positive Radon measure for which

$$
\left(\mu_{0}(\varphi) \mid \mu_{0}(\psi)\right)=v(\varphi \bar{\psi}) \text { for all } \varphi, \psi \in \mathscr{K}_{C}(\Gamma) .
$$

Then, $|\mathscr{F} f|^{2} \in \mathscr{L}^{1}(v)$ for all $f \in \mathscr{K}_{C}(G)$. By Theorem 2.5 the mapping $B: \mathscr{K}_{C}(G) \times$ $\mathscr{K}_{C}(G) \rightarrow C$,

$$
B(f, \bar{g})=(\mu(f) \mid \mu(g))=\int \mathscr{F} f \overline{\mathscr{F}} g d v, \quad f, g \in \mathscr{K}_{C}(G),
$$

is then a positive definite translation invariant bimeasure, which clearly implies that $\mu$ is a stationary vector measure.

On the other hand, suppose $\mu: \mathscr{K}_{C}(G) \rightarrow H$ is a stationary vector measure. Let $B: \mathscr{K}_{C}(G) \times \mathscr{K}_{C}(G) \rightarrow C$ be the (positive definite translation invariant) bimeasure defined by $\mu$ and let $v: \mathscr{K}_{C}(\Gamma) \rightarrow C$ be the positive Radon measure for which

$$
\int(u, \bar{v}) d B=\int \mathscr{F} u \overline{\mathscr{F} v} d v \quad \text { for all } u, v \in \mathscr{L}^{1}(\mu) \cap \mathscr{L}^{1}(G)
$$

(cf. Theorem 2.5).

Put

$$
E=\left\{\int u d \mu \mid u \in \mathscr{L}^{1}(\mu) \cap \mathscr{L}^{1}(G)\right\} .
$$

Define a mapping $j: E \rightarrow L^{2}(v)$ by setting

Since

$$
j(x)=\mathscr{F} u, \quad \text { if } \quad x=\int u d \mu \quad \text { for some } \quad u \in \mathscr{L}^{1}(\mu) \cap \mathscr{L}^{1}(G) .
$$

$$
\left\|\int u d \mu\right\|^{2}=\int(u, \bar{u}) d B=\int|\mathscr{F} u|^{2} d v \quad \text { for all } \quad u \in \mathscr{L}^{1}(\mu) \cap \mathscr{L}^{1}(G),
$$

the mapping $j: E \rightarrow L^{2}(v)$ is a well-defined inner product preserving linear mapping. Since $E$ is a dense linear subspace in $\overline{\operatorname{sp}}\{\mu\}$ and since $L^{2}(v)$ is a complete normed space, the mapping $j: E \rightarrow L^{2}(v)$ can be extended, by continuity, to a uniquely determined inner product preserving linear mapping $J: \overline{\mathrm{sp}}\{\mu\} \rightarrow L^{2}(v)$. Furthermore, by Lemma 3.2, the linear span of the functions $\mathscr{F} u, u \in \mathscr{L}^{1}(\mu) \cap \mathscr{L}^{1}(G)$, is a dense linear subspace in $L^{2}(v)$. Thus, the mapping $J: \overline{\mathrm{sp}}\{\mu\} \rightarrow L^{2}(v)$ is a surjection; and it has an inner product preserving inverse $J^{-1}: L^{2}(v) \rightarrow \overline{\operatorname{sp}}\{\mu\}$.

Define an orthogonally scattered vector measure $\mu^{\prime}: \mathscr{K}_{C}(\Gamma) \rightarrow L^{2}(v)$ by $\mu^{\prime}(\varphi)=\varphi$, $\varphi \in \mathscr{K}_{C}(\Gamma)$ (cf. Lemma 3.1). Since, by Theorem 2.5, $\mid \mathscr{F} u^{2} \in \mathscr{L}^{1}(v)$ for all $u \in \mathscr{L}^{1}(\mu) \cap$ $\mathscr{L}^{1}(G)$, we get by Lemma 3.1 that $\mathscr{F} u \in \mathscr{L}^{1}\left(\mu^{\prime}\right)$ and

$$
\int \mathscr{F} u d \mu^{\prime}=\mathscr{F} u \quad \text { for all } u \in \mathscr{L}^{1}(\mu) \cap \mathscr{L}^{1}(G) .
$$

Since the mapping $J^{-1}: L^{2}(v) \rightarrow \overline{\mathrm{sp}}\{\mu\}$ is an inner product preserving linear mapping, the vector measure $\mu_{0}: \mathscr{K}_{C}(\Gamma) \rightarrow \overline{\mathrm{sp}}\{\mu\}$, defined as $\mu_{0}=J^{-1} \circ \mu^{\prime}$, is orthogonally scattered and $\left(\mu_{0}(\varphi) \mid \mu_{0}(\psi)\right)=v(\varphi \bar{\psi})$ for all $\varphi, \psi \in \mathscr{K}_{C}(\Gamma)$. Thus, $\mathscr{L}^{1}\left(\mu_{0}\right)=$ $\mathscr{L}^{2}(v)$ and

$$
\left(\int u d \mu_{0} \mid \int v d \mu_{0}\right)=\int u \bar{v} d v \quad \text { for all } u, v \in \mathscr{L}^{2}(v)
$$


especially for all bounded Borel functions $u: \Gamma \rightarrow C, v: \Gamma \rightarrow C$ with compact support. Furthermore, for all $u \in \mathscr{L}^{1}(\mu) \cap \mathscr{L}^{1}(G)$; especially for all bounded Borel functions $u: G \rightarrow C$ with compact support

$$
\int u d \mu=J^{-1}(\mathscr{F} u)=J^{-1}\left(\int \mathscr{F} u d \mu^{\prime}\right)=\int \mathscr{F} u d\left(J^{-1} \circ \mu^{\prime}\right)=\int \mathscr{F} u d \mu_{0} .
$$

Finally, it follows from Lemma 3.1 that $\overline{\mathrm{sp}}\left\{\mu^{\prime}\right\}=L^{2}(v)$. Since the mapping $J^{-1}: L^{2}(v) \rightarrow \overline{\mathrm{sp}}\{\mu\}$ is an inner product preserving surjection, it follows that

$$
\overline{\mathrm{sp}}\left\{\mu_{0}\right\}=\overline{J^{-1}\left(\operatorname{sp}\left\{\mu^{\prime}\right\}\right)}=\overline{\mathrm{sp}}\{\mu\} .
$$

We still prove the uniqueness of the orthogonally scattered vector measure $\mu_{0}: \mathscr{K}_{C}(\Gamma) \rightarrow \overline{\mathrm{sp}}\{\mu\}$ satisfying (3.2).

Let $\mu_{0}: \mathscr{K}_{C}(\Gamma) \rightarrow \overline{\mathrm{sp}}\{\mu\}$ and $\mu_{0}^{\prime}: \mathscr{K}_{C}(\Gamma) \rightarrow \overline{\mathrm{sp}}\{\mu\}$ be two orthogonally scattered vector measures such that

$$
\mu(f)=\int \mathscr{F} f d \mu_{0}=\int \mathscr{F} f d \mu_{0}^{\prime} \text { for all } f \in \mathscr{K}_{C}(G) .
$$

Furthermore, let $v: \mathscr{K}_{C}(\Gamma) \rightarrow C$ and $v^{\prime}: \mathscr{K}_{C}(\Gamma) \rightarrow C$ be the positive Radon measures for which

$$
\left(\mu_{0}(\varphi) \mid \mu_{0}(\psi)\right)=v(\varphi \bar{\psi}) \quad \text { and } \quad\left(\mu_{0}^{\prime}(\varphi) \mid \mu_{0}^{\prime}(\psi)\right)=v^{\prime}(\varphi \bar{\psi}), \quad \varphi, \psi \in \mathscr{K}_{C}(\Gamma) .
$$

It then follows that

$$
B(f, \bar{g})=\int \mathscr{F} f \overline{\mathscr{F} g} d v=\int \mathscr{F} f \overline{\mathscr{F} g} d v^{\prime} \text { for all } f, g \in \mathscr{K}_{C}(G) .
$$

Thus, by Theorem 2.5, $v=v^{\prime}$. Therefore, $\mathscr{L}^{1}\left(\mu_{0}\right)=\mathscr{L}^{1}\left(\mu_{0}^{\prime}\right)$ and

$$
\left\|\int u d \mu_{0}\right\|=\left\|\int u d \mu_{0}^{\prime}\right\| \text { for all } u \in \mathscr{L}^{1}\left(\mu_{0}\right) \text {. }
$$

Let $\varphi \in \mathscr{K}_{C}(\Gamma)$; and let $\varepsilon>0$ be given. Since, by Lemma 3.2, the set $\left\{\mathscr{F}_{f} \mid f \in \mathscr{K}_{C}(G)\right\}$ is dense in $L^{2}(v)$, there exists an $f \in \mathscr{K}_{C}(G)$ such that

$$
\left(\int|\mathscr{F} f-\varphi|^{2} d v\right)^{1 / 2}=\left\|\int(\mathscr{F} f-\varphi) d \mu_{0}\right\|=\left\|\int(\widetilde{F} f-\varphi) d \mu_{0}^{\prime}\right\|<\varepsilon .
$$

Therefore,

$$
\left\|\int \varphi d \mu_{0}-\int \varphi d \mu_{0}^{\prime}\right\| \leqq\left\|\int(\mathscr{F} f-\varphi) d \mu_{0}\right\|+\left\|\int(\mathscr{F} f-\varphi) d \mu_{0}^{\prime}\right\|<2 \varepsilon
$$

which implies that $\mu_{0}$ and $\mu_{0}^{\prime}$ are identical.

The theorem is proved.

Remark. The mapping $J^{-1}: \overline{\mathrm{sp}}\{\mu\} \rightarrow L^{2}(v)$, defined in the proof of Theorem 3.3, is exactly the same as the mapping $\mathscr{F}: \mathscr{H}_{\mu} \rightarrow L^{2}(\breve{\mu})$ defined in Theorem 5.1 by Argabright and Gil de Lamadrid [1]. Theorem 3.3 thus serves a new interpretation of this theorem which is essentially due to Godement [10; p. 76], [11].

The following theorem is a direct consequence of Theorems 3.3, 2.7 and 2.8. 
Theorem 3.4. Let $\mu: \mathscr{K}_{C}(G) \rightarrow H$ be a stationary vector measure and let $\mu_{0}: \mathscr{K}_{C}(\Gamma) \rightarrow \overline{\mathrm{sp}}\{\mu\}$ be the uniquely determined orthogonally scattered vector measure satisfying (3.2). Then:

(i) $\mu_{0}$ is bounded, if and only if there exists a constant $c>0$ such that

$$
\|\mu(f)\|^{2} \leqq c \sup |\mathscr{F} f|^{2} \text { for all } f \in \mathscr{K}_{C}(G) .
$$

If (3.3) holds, then $\mathscr{L}^{1}(G) \subset \mathscr{L}^{1}(\mu)$ and

$$
\int u d \mu=\int \mathscr{F} u d \mu_{0} \text { for all } u \in \mathscr{L}^{1}(G) \text {. }
$$

(ii) If there exist constants $c>0, c^{\prime}>0$ such that

$$
\begin{aligned}
\|\mu(f)\|^{2} & \leqq c \int|f|^{2} d \lambda, \quad f \in \mathscr{K}_{C}(G) ; \\
\left\|\mu_{0}(\varphi)\right\|^{2} & \leqq c^{\prime} \int|\varphi|^{2} d \theta, \quad \varphi \in \mathscr{K}_{C}(\Gamma),
\end{aligned}
$$

then $\mathscr{L}^{2}(G) \subset \mathscr{L}^{1}(\mu), \mathscr{L}^{2}(\Gamma) \subset \mathscr{L}^{1}\left(\mu_{0}\right)$ and

$$
\int u d \mu=\int \mathscr{F} u d \mu_{0} \text { for all } u \in \mathscr{L}^{2}(G) \text {. }
$$

We close this paper by recalling an example due to Masani [14; pp. 92-94].

Example 3.5. Suppose $\mu: \mathscr{K}_{C}(G) \rightarrow H$ is a stationary vector measure which is also orthogonally scattered. There then exist positive Radon measures $v_{0}: \mathscr{K}_{C}(G) \rightarrow C$ and $v: \mathscr{K}_{C}(\Gamma) \rightarrow C$ such that for all $f, g \in \mathscr{K}_{C}(G)$

$$
(\mu(f) \mid \mu(g))=v_{0}(f \bar{g})=\int \mathscr{F} f \overline{\mathscr{F} g} d v .
$$

Since $\mu$ is stationary, the Radon measure $v_{0}$ is translation invariant, a fortiori, there exists a constant $c>0$ such that $v_{0}=c \lambda$. (If $\mu=0$, then $c=0$.) It is clear that $v$ is the Plancherel measure corresponding to $v_{0}$. Thus, there exists a constant $c^{\prime}>0$ such that $v=c^{\prime} \theta$. (If $\mu=0$, then $c^{\prime}=0$.)

Finally, we note that the condition (ii) stated in Theorem 3.4 is satisfied for $u$.

On the other hand, it is clear that any vector measure $\mu: \mathscr{K}_{C}(G) \rightarrow H$ for which there exists a constant $c \geqq 0$ such that

$$
(\mu(f) \mid \mu(g))=c \int f \bar{g} d \lambda, \quad f, g \in \mathscr{K}_{C}(G),
$$

is orthogonally scattered and stationary.

Acknowledgement. The first draft of this paper was written during the author's stay at the Department of Probability and Statistics of the University of Sheffield in the spring and summer terms of 1977. The work has been supported by the Royal Society. The author wants to express his thanks to Professor C. Berg for his valuable remarks concerning Theorem 2.5 and Example 2.6.

\section{References}

[1] Argabright, L., and J. GiL De LAmAdrid: Fourier analysis of unbounded measures on locally compact abelian groups. - Mem. Amer. Math. Soc. 145, 1974, 1-53.

[2] Aronszajn, N.: Theory of reproducing kernels. - Trans. Amer. Math. Soc. 68, 1950, 337404. 
[3] Benedetto, J. J.: Spectral synthesis. - Mathematische Leitfäden, B. G. Teubner, Stuttgart, 1975.

[4] Berg, C.: Sur les semi-groupes de convolution. - Théorie du potentiel et analyse harmonique. Ed.: J. Faraut. Lecture Notes in Mathematics 404, pp. 1-26, Springer-Verlag, Berlin-Heidelberg-New York, 1974.

[5] Berg, C.: Transformation de Fourier de mesures de type positif sur un groupe abélien localement compact. - Séminaire de théorie du potentiel, Paris 1972-1974. Réd.: F. Hirsch et G. Mokobodzki. Lecture Notes in Mathematics 518, pp. 37-44, Springer-Verlag, Berlin-Heidelberg-New York, 1976.

[6] Berg, C., and G. Forst: Potential theory on locally compact Abelian groups. - Ergebnisse der Mathematik und ihrer Grenzgebiete 87, Springer-Verlag, Berlin-HeidelbergNew York, 1975.

[7] Brillinger, D. R.: The spectral analysis of stationary interval functions. - Proceedings of the Sixth Berkeley Symposium on Mathematical Statistics and Probability. I, pp. 483-513, University of California Press, Berkeley-Los Angeles, 1972.

[8] Daley, D. J., and D. Vere-Jones: A summary of the theory of point processes. - Stochastic point processes: Statistical analysis, theory and applications, pp. 299-383. Ed.: P. A. W. Lewis. Wiley-Interscience, New York-London-Sydney-Toronto, 1972.

[9] Gelfand, I. M., and N. YA. Vilenkin: Generalized functions. Vol. 4, Applications of harmonic analysis. - Academic Press, London-New York, 1964 (English translation).

[10] Godement, R.: Sur la théorie des représentations unitaires. - Ann. of Math. 53, 1961, 69124.

[11] Godement, R.: Introduction aux travaux de A. Selberg. - Séminaire Bourbaki, 9e année, 1956/1957 [2e édition, corrigée]. Exposé No. 144. Secrétariat mathematique, Paris, 1959.

[12] Horváth, J.: Topological vector spaces and distributions, Vol. 1. - Addison-Wesley series in mathematics, Addison-Wesley Publishing Company, Reading-Palo Alto-London-Don Mills, 1966.

[13] Iтô, K.: Stationary random distributions. - Mem. Coll. Sci. Kyoto Imp. Univ. Ser. A 28, 1954, 209-223.

[14] MASANI, P.: Orthogonally scattered measures. - Advances in Math. 2, 1968, 61-117.

[15] NiEMI, H.: Stochastic processes as Fourier transforms of stochastic measures. - Ann. Acad. Sci. Fenn. Ser. A I 591, 1975, 1-47.

[16] NIEMI, H.: On the support of a bimeasure and orthogonally scattered vector measures. - Ann. Acad. Sci. Fenn. Ser. A I 1, 1975, 249-275.

[17] Rudin, W.: Fourier analysis on groups. - Interscience tracts in pure and applied mathematics 12, Interscience Publishers, New York-London, 1962.

[18] Thomas, E.: L'intégration par rapport à une mesure de Radon vectorielle. - Ann. Inst. Fourier (Grenoble) 20:2, 1970, 55-191.

\author{
University of Helsinki \\ Department of Mathematics \\ SF-00100 Helsinki 10 \\ Finland
}

Received 13 November 1978 